\title{
Analytical binary black hole model of Sgr A* and its implications
}

\author{
Tapan K. Chatterjee \\ University of the Americas, Department of Physics, A. P. 1316, Puebla, Mexico \\ e-mail: chtapan@yahoo.com
}

\begin{abstract}
Surrounding the Galactic center is a molecular cloud with a central black hole. This raises the question as to how the hole was formed. While clouds containing HCN are circulating around the Galactic center, they do not all move at the same speed and so collide mutually and should make a more uniform motion and distribution after a period of some 100 kyrs (e.g., Ekers, van Gorkom, Schwartz et al. 1983). Hence some very energetic phenomena must have occurred within that period.
\end{abstract}

Keywords. Black hole physics - galaxies: nuclei

\section{Introduction and Theory}

The radio source at the Galactic center, $\operatorname{Sgr} \mathrm{A}^{*}$, is an excellent candidate for a black hole, as the flat sub-mm spectrum with synchrotron emission requires high compactness (e.g., Zylka, Mezger, Warg-Thompson et al. 1995; Duschl \& Lesch 1994). However, its radiation intensity is orders of magnitude weaker. Several observations indicate nonhomogeneous source structure: different core sizes at different $\mathrm{mm}$ wavelengths; different variability at sub-mm and radio wavelengths; varying simultaneous spectral indices (e.g., Lacy, Townes \& Hoolenbach 1982). The radial velocities of the molecular hydrogen emission regions, straddling the Galactic center, exhibit an asymmetry between the two peaks, corresponding to a radial velocity component of $40 \mathrm{~km} / \mathrm{s}$, with respect to the Sun (Clube 1986). No such effect is noticeable from the distribution of radial velocities of the stars around the Galactic center. This indicates rotational motion with practically no offset in radial velocity (e.g., Kent 1992). An interpretation of the radial asymmetry is that it is due to the orbital motion around the black hole.

\section{Results and Conclusions}

In this context we model analytically a binary black hole (Magalinsky \& Chatterjee 2002). Using the variational principle (adopted from the semi-classical approach) a criterion is formulated (which tends towards energy minimization and gravity softening) to study the orbital motion of the pair. An analysis of the expected distribution of orbital parameters, on the basis of statistical mechanics, indicates that the system is characterized by a state preferring high eccentricities.

The system is found to slowly circularize its orbit in the adiabatic regimen, but in a large timescale. This has been confirmed by numerical simulations (Chatterjee \& Magalinsky 2002). Thus the separation of the black holes decreases gradually, followed by coalescence after which new pairs of black holes are likely to be established. The system exhibits poor accretion efficiency, as the accretion rate varies radially (Chatterjee \& Magalinsky 2006). This is because most of the mass, from the stellar wind appears to 
fall in the center of mass of the system; and is not accreted by either component of the binary.

Preliminary results indicate that the model explains the low intensity of radiation of Sag A The binarity of a black hole system is very hard to confirm observationally. (only VLBI might detect radio radiation from both components). A recourse has to be taken to indirect methods. A regular pattern of variability of optical emission lines (Halpern \& Fillippenko 1988), the precessional behavior of radio jets, (Rees 1984) and the changing precessional period due to orbital motion of the binary (Roos 1988; Roos, Kaastra \& Hummel 1993) are expected.

\section{References}

Chatterjee, T. K. \& Magalinsky, V. B. 2002, Astron. Astrophys. Trans., 21, 79

Chatterjee, T. K. \& Magalinsky, V. B. 2006, Astron. Astrophys. Trans., submitted

Clube, S. V. M. 1986, The Observatory, 106, 166

Duschl, W. J. \& Lesch, H. 1994, A\&A, 286, 431

Ekers, R. D., van Gorkom, J. H., Schwartz, U. J. \& Goss, W. M. 1983, A\&A, 122, 143

Halpern, J. P. \& Fillippenko, A. V. 1988, Nature, 331, 46

Kent, S. M. 1992, ApJ, 387, 181

Lacy, J. H., Townes, C. H. \& Hoolenbach, D. J. 1982, ApJ, 262, 120

Magalinsky, V. B. \& Chatterjee, T. K. 2002, Astron. Astrophys. Trans., 18, 807

Rees, M. J. 1984, ARA\&A, 22, 123

Roos, N. 1988, ApJ, 334, 95

Roos, N., Kaastra, J. S. \& Hummel, C. A. 1993, ApJ, 409, 130

Zylka, R., Mezger, P. G., Warg-Thompson, D., Duschl, W. \& Lesch, H. 1995, A\&A, 297, 83 